\title{
VALORES GENERALES EN UNA MUESTRA DE ADOLESCENTES MEDIOS DE LA CIUDAD DE SANTO
} DOMINGO

Francia Reynoso*

RESUMEN

La adolescencia es una etapa de la vida que es parte del contínuum, se inicia según la OMS a los 10 años y termina a los 20 . Se subclasifica en adolescencia temprana, media.y tardía y cada uno de estos apartados tiene sus conductas propias y sus conductas esperadas.

En el discurrir de la misma se producen cambios en el orden físico, sicológico y social, pero de manera importante se enfatiza la construcción de su identidad y se afianzan, construyen y reformulan sus sistemas axiológicos.

En esta investigación hemos trabajado con una muestra de 300 adolescentes, de entre 14 y 17 años de edad, de distintos estratos sociales de la ciudad de Santo Domingo, con la intención de conocer sus formas de valorar y el qué valoran.

Trabajos clásicos como los de Scheler, Hartman, Frondizi nos han servido de marco conceptual para comparar sus postulados con nuestros hallazgos.

El punto de partida ha sido el de reconocer el valor como lo que vale y no de partir de la predeterminación de los valores como condición para investigar. Lo que ellos y ellas han considerado como válido, como estimable es lo que hemos considerado como valor y no lo que tradicionalmente se ha conocido como tal y que en la mayoría de los casos es referido al valor moral.

PALABRAS CLAVE

Valores, adolescencia

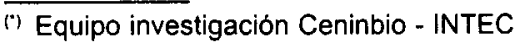




\subsection{ANTECEDENTES.}

A partir de 1992 nuestro país dio inicio a su preocupación por la temática adolescencia desde una perspectiva denominada de integralidad. Esta visión plantea la ubicación etaria entre los 10 y 20 años asumiendo todas sus dimensiones como parte del desarrollo, lo que define el perfil integral de los mismos.

Se dio una ruptura con los tradicionales enfoques que colocaban a la adolescencia como una etapa de transición a la adultez pero sin estatus propio, sino más bien como una etapa que debía conducir necesariamente a una crisis y rebeldía contra todo lo que significara autoridad.

Sus antecedentes procedían de visiones y estereotipos construidos en otras latitudes donde los cánones signados por autores clásicos daban un marco teórico que nos planteaban como conductas esperadas fenómenos tan conocidos en la literatura como el complejo de Edipo, por citar uno importante o conductas reactivas contra la sociedad y la familia.

Uno de los méritos de la nueva visión integral de la adolescencia le reubica históricamente, encuentra sus raíces en el siglo 18 con el advenimiento de la escuela y la pediatría rompiendo los modelos griegos que la ignoraban como etapa y que cifraban la madurez y la perfección en la juventud, que hoy sería denominada edad madura .

Gracias a este recorrido significativo de cambios en la historia hoy podemos comprender la adolescencia como una etapa más de la vida, como parte del desarrollo o contínuum vital y sobre todo conocer una reclasificación interna que coloca un primer estadio entre los 10 y 13 años conocido como adolescencia temprana a la cual pertenecen unas características específicas y unas conductas esperadas para la edad.

El segundo estadio es conocido como adolescencia media y está comprendida entre los 14 y 17 años de edad y es la pobla- 
ción objetivo de esta investigación por la que dedicaremos más espacio para su conocimiento. Finalmerte está la adolescencia tardía que se refiere al grupo comprendido entre los 17 y 20 años de edad.

\subsection{LA ADOLESCENCIA}

La adolescencia como tal es un proceso de maduración y desarrollo que abarca casi una década de la vida. Es una época de grandes cambios en todas las esferas que se desencadenan a partir de la pubertad.

Se trata de un período de gran vulnerabilidad, grandes oportunidades y avances tanto físicos como intelectuales, morales y sociales. Para su mejor comprensión la OMS divide la adolescencia en las 3 etapas ya mercionadas

En este artículo profundizaremos sobre la adolescencia media por ser el sujeto de nuestra investigación. Los aspectos biológicos sicológicos y sociales adquieren características muy propias las cuales pasaremos a reseñar para poder colocar nuestra propuesta axiológica.

Como hemos señalado antes la adolescencia media se ubica entre los 14 y 17 años y es una etapa donde se producen cambios notables en la esfera biológica. Para la hembras aparecen las mamas, pelo púbico/axilar, el cuerpo va adquiriendo formas cada vez más cercanas a la de los adultos. El punto clave de la biología en la adolescencia media en las hembras es la menarca o primera menstruación y la eyacularquia o primera eyaculación en el varón. Es la evidencia del inicio de su capacidad reproductiva.

En el aspecto sicológico se consolida la identidad sexual y se alcanza el pensamiento lógico formal, el pensamiento reflexivo que los acerca más a los adultos que los rodean. Erickson define la identidad como: 
“...un sentimiento vigorizante y subjetivo de mismidad y continuidad" 1

donde ellos alcanzan un sentido de autonomía y de tomar sus propias decisiones casi sin darse cuenta. El adolescente se ubica en una nueva dimensión temporal muy distinta a la de la infancia, el futuro comienza a reclamar su atención.

"El tiempo es una pieza fundamental de un gran rompecabezas: el de la integración de la identidad" 2

El sentimiento de identidad no comienza ni termina en la adolescencia. Se da una interrelación de los sistemas del pensamiento y los afectivos con una vida social que se amplía y que repercute en su maduración; el adolescente construye poco a poco su imagen y concepto de sí mismo, hasta que pueda reconocerse como una entidad separada y distinta de su entorno.

El comportamiento de los adolescentes en esta etapa está marcado por grandes cambios: demuestran gran creatividad, que expresan por medio de la música, el arte, poesía, discuten con su grupo de pares sobre moral, religión, ética, labores humanitarias, hay un predominio de los ideales nobles y asumen su ejecución como un compromiso personal. Lo mismo podriamos decir si son inducidos por caminos negadores de estos. Son vehementes con las ideas que defienden y fieles con las prácticas de lo que valoran.

En la adolescencia media se realiza la integración con la sociedad. En este momento los adolescentes tienen fuerza personal y no solo grupal.

Empiezan a mezclar valores de fuentes diversas con sus propios valores personales, se ha establecido una nueva conciencia

Erickson, Erick, 1974. Identidad, Juventud y Crisis.

2 Douvan y Adelson, 1980, Handbook on Adolescence. J. Willey y Sons 
o "súper yo" que debe ser capaz de cambiar, crecer y acomodarse ante las nuevas situaciones de la vida.

En lo relativo a la vida sexual en esta etapa los adolescentes no parecen responder solo, ni quizás principalmente, a motivaciones estrictamente sexuales y por así decir, intrínsecas. A veces estas se instigan por incitaciones externas, de ahí que los grupos de pares o amigos de edades similares jueguen un papel importante en el proceso de socialización de su sexualidad.

Pautas y guiones culturales gobiernan los rituales de interacción que culminan en la actividad sexual. Los prototipos y símbolos sexuales imperantes en una cultura determinan profundamente los contenidos, los modos y los significados de la conducta sexual.

Las expectativas sociales acerca de lo que el joven o la joven ha de hacer en tal o cual situación, por privada e interna que sea la escena, definen mucho el curso de su actividad sexual.

Para muchas adolescentes, además la intimidad sexual es una experiencia generadora de autonomía, de sentimiento de identidad, de autovaloración propia como persona atractiva y de toma de conciencia de su capacidad para la relación con otros.

La sexualidad adolescente es búsqueda de significado y está cargada de ricas connotaciones simbólicas. Podemos decir que la construcción de identidad, la definición de su marco axiológico y el desarrollo y énfasis de su sexualidad son puntos claves de este momento de la vida del adolescente medio.

\subsection{ANTECEDENTES DE INVESTIGACIONES SOBRE ADOLESCENCIA Y VALORES.EN REPÚBLICA DOMINICANA}

En la década de los 90 en nuestro pais se realizaron varias investigaciones relativa al mundo de los valores y la adolescencia. Podemos destacar los trabajos de Alvaro Tamayo y Migue! 
Suazo y luego la de Suazo y Valeirón entre otros, donde registraban valores sexuales en adolescentes dominicanos. En $2002 \mathrm{Mi}-$ guel Suazo con el equipo de investigación del Ceninbio investigamos de nuevo sobre valores sexuales pero en adolescentes tardíos de nivel universitario.

En esta ocasión hemos realizado esta investigación sobre valores generales en adolescentes medios a través del Centro INTEC de Bioética (Ceninbio), junto al equipo de investigaciones del Ceninbio.

\subsection{INTRODUCCIÓN.}

Nuestra cultura occidental al estudiar la juventud se ha caracterizado entre otras cosas por establecer diferencias entre los adolescentes y el mundo adulto. en nombre de "choques generacionales", "diferencias de época" "cambios de los tiempos", entre otros.

Nuestro país y cultura nacional no ha escapado a este pensamiento y hemos contribuido sobremanera a crear una diferenciación entre los adolescentes ubicándoles en la ambivalencia de ser demasiado grandes para ser niños y demasiado pequeños para ser adultos.

En el área médica ninguna especialidad quiere reconocerlos como propios de su competencia, ya que el pediatra los encuentra muy grandes y el internista muy pequeños.

La moderna reconceptualización que de esta etapa se realiza reubica dentro de la normalidad su desarrollo y establece una serie de parámetros y normas que les son propios y que hasta ahora les han sido desconocidos. Tal es el caso del sistema axiológico o su mundo de valores en el cual sustentan sus prácticas y desarrollo vital. 
En esta presentación abordamos la relación de los adolescentes a partir de su reconceptualización y del sistema de valores en que se desenvuelven.

\subsection{ADOLESCENCIA Y VALORES.}

Para algunos la adolescencia ha sido definida como época de turbulencia y tiempo de rebeldía. Ya en el siglo 17 los Alemanes hablaban del "Sturm und drang" o sea "Tormenta y stress" para definir los supuestos ejes en los que se movía la adolescencia y distintos autores se han pasado absolutizando estos conceptos y lógicamente creando un marco referencial que permite hacer una taxonomía restrictiva de la juventud.

Para estos los adolescentes como tal no son definidos como un ente social establecido sino como una transición amorfa, dependiente del poder del mundo adulto. El concepto de la adolescencia es relativamente nuevo en la historia de la humanidad. Su etimología proviene del latín adolescere que quiere decir crecer.

La literatura producida en países desarrollados ha dado las definiciones propias de su contexto llegando a asumirlas como válidas en el tercer mundo creando una distorsión, ya que esos mandatos no necesariamente se aplican de manera absoluta.

Hoy se ha reconceptualizado la adolescencia desde nuevos contextos de una manera especial. Ha sido clasificada en : temprana, media y tardía dependiendo de si las edades se encuentran entre los 10-13; 14-16; 17-20 años, acogiéndonos a los rangos aportados por la Organización Mundial de la Salud, así como desde la perspectiva del impacto que le confieren los denominados contextos de desarrollo donde se producen.

Ya antes señalamos que la etapa de la adolescencia se caracteriza por ser momento de construcción de identidad y ello se hace desde un contexto axiológico determinado. Los valores pro- 
venientes de la familia, la escuela, la iglesia y demás medios de socialización sirven de marco para la búsqueda de ese perfil.

Tradicionalmente se acusa a los jóvenes y en especial a los adolescentes medios de ser responsables de una supuesta pérdida de valores, esto debido a que en la práctica no ratifican muchos de los valores adultos en su versión originaria sino que por el contrario tienen propuestas más dinámicas que en muchas ocasiones no niegan la intención de estos.

El mayor problema de los valores es que las generaciones adultas los perciben como entes inamovibles que deben repetirse en la juventud pero no las desligan de los contextos en que ellos las aprendieron y aspiran a que los valores se reproduzcan y los contextos también lo cual resulta imposible y genera el conflicto entre las generaciones.

Negamos la idea de choque generacional per se ya que lo que se confronta en realidad es la intención de reproducir épocas y modelos axiológicos descontextualizados.

En muchos otros casos se ha hablado de valores como algo establecido, inamovible y permeado por las regulaciones de la moral imperante. El valor ha estado indisolublemente vinculado al poder en cualquiera de las instancias en que se desarrolle. Cuando normamos en nuestras casas, lo hacemos desde la perspectiva de padres, en la escuela como autoridad, en la iglesia, en los clubes, etc y en el país a través de los mecanismos de acción del poder.

Determinados valores fueron llamado "Universales" expresando su representatividad e inamovilidad. Nadie osaría cuestionar si el amor es un valor universal, ya que hasta quienes van a la guerra y matan dicen hacerlo por amor, a la patria, a los principios, a los derechos. Otros son menos generales y llevan en su interior el dinamismo que permite su transformación en el tiempo y se les denomina valores particulares. 
Hoy se dice que los valores son principios, propiedades y creencias a los cuales les damos un grado de significación. Para Risieri Frondizi "los valores no son, por consiguiente, ni cosas, ni vivencias, ni esencias: son valores" "Los valores no son cosas, ni elementos de cosas, sino propiedades, cualidades sui generis, que poseen ciertos objetos llamados bienes" 3

Esta primera discusión diferencia a los valores de las cosas y de los hechos. Los valores pasan por los hechos pero se diferencian de estos. Al respecto Diego Gracia afirma:

“...además de hechos hay valores. Y si los hechos tienen la característica de ser, bien datos de percepción, bien construcciones intelectuales basados directamente sobre datos perceptivos, los valores tienen la peculiaridad de no ser directamente perceptivos" 4 .

Los valores se estiman más que decir que se perciben.

Estas acepciones implican establecer que los valores como tal no se pierden sino que cambian, se transforman, pero que como son expresión de una relación de poder pueden, y en efecto lo hacen, encontrar fuertes resistencias en la práctica, en los hechos, para ser aceptados o cambiados, en la medida que afecten los intereses de sectores dados.

Ha sido mucho el debate en la historia sobre los valores para poder situarlos. Para unos prima su carácter subjetivo sobre el objetivo y para otros lo contrario. Desde la ética de Scheler y Hartman se colocan desde el objetivismo, "se sostiene además, que los valores se aprehenden emocionalmente, es decir (en la terminología de Scheler) por medio de actos emocionales intencionales, como el "percibir sentimental" (Fuhlen), el "preferir" (Vorziehen) o el "amar" (Lieben) ${ }^{5}$

3 Frondizi R: " Qué son los valores? ". Breviarios Fondo de Cultura Económica, México p. $17 ; 1995$

4 Gracia D: "Etica de la calidad de la vida". Cuadernos del Programa Regional de Bioética, OPS.OMS. No. 2, Chile p.45; 1996.

5 Maliandi R: "Notas para una rehabilitación de la Etica material de los valores" Escritos de filosofia, Buenos Aires. No. 21-22 p. 70; 1992 
Otros debatieron desde el ángulo de definición de las valoraciones correctas e incorrectas. Como vemos no hay un consenso total en su definición pero sí hay cierta claridad que nos permite movernos y afiliarnos a alguno de los polos.

Desde estas cosmovisiones se refleja que en los adolescentes no se ha reconocido un sistema de valores propios y que siempre fueron referidos al sistema de valores de los adultos que son símbolo de autoridad (padres, maestros, religiosos...) descartando la originalidad y posibilidad de que ellos a la vez que asumen valores familiares, también construyan los propios, acordes con su historia personal e interacción con su medio social, que es en última instancia su contexto histórico.

Esta construcción guarda una relación directa con los niveles de autoestima con que estos hayan sido alimentados en su proceso de crecimiento desde la niñez.

"Todos nosotros necesitamos el reconocimiento de nuestro valor. Yo tengo mi valor y otro tiene su valor, pero el adolescente necesita fundamentalmente saber qué los otros piensan de él, y que él se siente bien como persona (con su cuerpo, sus afectos, sus ideas)" 6

La autoestima será construida a través de las relaciones afectivas que se desarrollan en el ámbito familiar, escolar y de los demás medios de socialización y serán el reflejo del marco conceptual de sus implementadores en cuanto a lo que es el proceso de desarrollo y la construcción de los valores propios y los transmitidos .

Cuando los adultos hacemos comparaciones como éstas "en mi tiempo eso no se veía", "en mi época había respeto", "la música de ahora no sirve", "las modas de hoy son un disparate" y otras, estamos en realidad torpedeando los nuevos sistemas de valores, de preferencias, y a la vez haciendo una resistencia al

6 Donas S: "Marco epidemiológico conceptual de la salud integral del adolescente" Versión revisada y ampliada noviembre 1997, OPS. Costa Rica p.3; 1997. 
paso de las nuevas generaciones, intentando perpetuarnos en la historia como único punto de referencia y solidificando las relaciones de poder que descansan en el mantenimiento de los sistemas de valores anteriores.

El reconocimiento de que la adolescencia es un tiempo de creación de identidad y de separatividad en la cual el joven crea su propia personalidad, plantea un reto que implica de alguna manera un desplazamiento real de las generaciones que tienen el poder y el mando en las instancias donde ellos se desarrollan. Es el caso de los padres en primera instancia y aceptarlo así es reconocer sus limitaciones y temporalidad, por lo que algunos apelan a confrontar su autoridad con la naciente autonomización del joven, develando las relaciones de poder que allí residen.

El primer conflicto encontrado viene dado por el cambio sufrido en la transmisión de los valores de padres a hijos. Un ambiente más permisivo, la amplitud de los canales de información de los medios de comunicación social, la tecnología, la comunicación inter-países y culturas son responsables en gran parte de estos cambios. La educación formal e informal ha jugado la otra parte con su apertura hacía la formación de personas, con capacidad de elegir, de discernir y estimar. Más dificil aún cuando, por demás, lo que se evalúa son los comportamientos y sustentos axiológicos de la sexualidad.

En este sentido se inscribe el objeto de este trabajo. En intentar conocer el sistema de valores generales subyacentes en las y los adolescentes dominicanos que participaron.

No todo lo que se estima como bueno o válido podrá ser considerado como valor. De ser así privilegiaríamos de manera absoluta e incuestionable la subjetividad del valor cayendo en absolutizaciones imposibles de legitimar.

Para evitar estas situaciones partimos del reconocimiento de la bipolaridad del valor. Los valores como tal se presentan de manera polar con un doble componente el positivo y el negativo 
ambos con existencia propia, propiciando más aún la confusión al hacer su aparición en determinados escenarios como el de la adolescencia, que ya de por sí trae sus propias distorsiones en su conceptualización y con la sexualidad que arrastra tantos estigmas por su historia propia.

Los valores son bipolares y por demás guardan una relación jerárquica que es en última instancia en la que las personas experimentamos mayores diferencias al ordenar nuestros sistemas, validarlos como buenos y proponerlos como instancia de confrontación de los sistemas de los demás. El orden está determinado por las preferencias y estimaciones. Se coloca en el orden superior el más estimado "aunque a veces elija el inferior por razones circunstanciales" señala Frondizi. La polaridad enfrenta a su componentes como positivo y negativo y la ética se encargará de que se realicen los primeros y se rechacen los segundos, pero los estudia a ambos.

Scheler planteará que la tarea será realizar los valores positivos y no solamente el ser bueno, los valores no solo se definen, sino que se realizan a través de su aprehensión. Esa es la tarea de la ética, hacer valores positivos.

Esta relación explica por qué no puede definirse una tabla de valores con los cuales confrontarnos, ya que en ella confluyen las individualidades y los contextos de los sujetos que las implementan y las épocas y culturas en que se desenvuelven los actores, surgiendo así tantos ordenamientos como personas y como momentos de su vida.

"Sin embargo, la existencia de un orden jerárquico es una incitación permanente a la acción creadora y a la elevación moral. El sentido creador y ascendente de la vida se basa, fundamentalmente en la formación del valor positivo frente al negativo y del valor superior frente al inferior" 7

$\overline{7}$ Frondizi. Op. cit. p. 27 
En los y las adolescentes sucede lo mismo: construyen, recrean, ordenan y jerarquizan en base a su mundo de aprendizaje y a sus contextos de desarrollo, lo cual les coloca en desventaja en una sociedad marcada por el autoritarismo adulto que valida sus experiencias pasadas como únicas validadas. Si bien esto es incorrecto también lo será el validar como bueno todo lo que provenga de los adolescentes por ser fruto de su época. La moralidad del valor no tiene tiempo, lo que cambian son sus formas de expresión.

Tanto la valoración adolescente como la propia adolescencia es enjuiciada desde el mundo adulto con una perspectiva de negatividad.

Éste no es un resultado del azar, hay todo un marco teórico conceptual implícito que recoge una visión negativa de esta etapa y que considera que en ella faltan elementos emocionales, de madurez y de responsabilidad por lo que urge salir de ella de la mejor manera posible. En determinados sectores adultos se llega a visualizar este período de la vida como un constante adolescer. No tenemos a mano datos escritos que avalen estas afirmaciones, pero en la cotidianidad familiar y escolar, así como en la prensa, aparece como una constante.

Se enfrentan posiciones y las sustentan poderes. Los y las adolescentes con sus vivencias, respuestas y conductas esperadas, versus el mundo adulto que detenta el poder en todas sus manifestaciones y que en su mayoría asume la posición de reconocer la adolescencia como etapa de crisis per se.

El objetivo principal de este trabajo reside en poder ubicar la adolescencia como una etapa normal del desarrollo de la vida, rescatándola del contexto histórico que ha dado pies a malos entendidos sobre su definición y sobre todo en lo referente a su abordaje

Uno de los aspectos más mal tratado ha sido probablemente el referido al contexto axiológico donde afirmaciones mal enfo- 
cadas han resaltado una supuesta pérdida de valores en la sociedad y atribuye de manera preferencial la responsabilidad a las actuales generaciones. Ser joven ha llegado a ser sinónimo de inmadurez y de irresponsabilidad para muchos.

La constante establecida de entender la adolescencia como un puente entre la niñez y la adultez ha implicado también la de precisar la necesidad de saltar u obviar ese puente por ser un indicador ineludible de crisis, rebeldía o como antes llamaron los alemanes de "tormenta y stress". Múltiples definiciones la sitúan como etapa intermedia, de transición, puente, para simbolizar que ella no es en sí misma, sino que es vía para alcanzar etapas de mayor garantía emocional y solidez.

La sicología clásica, en especial de procedencia anglosajona, definió y conceptualizó a sus adolescentes desde sus contextos de desarrollo, pero países como el nuestro, con escaso desarrollo científico y generación de conocimientos, asumió esas teorías como propias sin darles el contexto preciso, de ahí que justificara la percepción de la misma como una patología . Es correcta la visión que la relaciona en función de sus contextos, épocas, culturas y valoraciones, lo incorrecto es pretender universalizar el concepto sin tomar en cuenta esas mediaciones.

Los modelos sociales como los norteamericanos nos han sido ofrecidos como espejo para educar y orientar a nuestra adolescencia desde el ámbito escolar y familiar, presentándonos sus experiencias de independencia a temprana edad, sus conductas a partir de estos hechos, como patrón a seguir, generándonos un nuevo conflicto que es el de generar expectativas sobre determinadas conductas globales que en nuestros contextos son patológicas. El proceso de independencia, por citar uno, se inicia tempranamente en esas culturas mientras nuestras raíces latinoamericanas nos hacen vivenciar y degustar del ámbito paterno sin límites de tiempo. 
Una definición como esta no satisface las expectativas nuestras si hacemos lecturas de sus autores y genera en nosotros un sentimiento conflictivo entre lo que debo esperar y lo que se registra. Al no haber coincidencia con lo esperado sino respuestas diferentes han habido interpretaciones patologizantes de la adolescencia.

¿De dónde proviene esa visión patológica de este estadio vital? Una detallada revisión requiere establecer como premisa principal que la vida es un contínuum al que hay que revisar desde la niñez para poder explicarnos por qué la adolescencia no apareció como fenómeno real en la historia sino hasta el siglo XVIII y por qué luego que aparece nace con el sello estigmatizante que le reconoce como época crucial de la que es recomendable salir cuanto antes y a la que además se le factura la responsabilidad de situaciones como una supuesta pérdida de los valores.

Los valores se definen como las cualidades, principios, normas y creencias que tenemos las personas y como tal les corresponde un orden y una jerarquía que les coloca una determinada importancia en el mundo individual de los sujetos y en el colectivo de las épocas.

En esta investigación hemos reunido las dos variables adolescencia y valores las cuales demuestran de qué manera se conjugan las mismas en la práctica. En esta primera entrega estamos presentando el marco referencial en que se desarrolla el contexto axiológico y en la segunda los resultados de los hallazgos.

\section{BIBLIOGRAFÍA}

1 Aristóteles. Retórica II 12:1389

2 Blanco, Juan Antonio. “Tercer Milenio". Edición del centro Félix Valera, La Habana, Cuba, 1998.

3 Bolívar, Antonio: "La evaluación de valores y actitudes", Grupo ANAYA, S. A., Madrid, España, 1998. 
4 Cairo, Valeirón y Zaiter. Sociedad, cultura e identidad en la República Dominicana. Tesis de maestría en sicología comunitaria. UASD. Santo Domingo 1998.

5 Cortina, Adela. El mundo de los valores, Editorial Codice Ltda, Bogotá, 2000.

6 De Láncer, Víctor Hugo. "Sistema Educación y Cultura Democrática". Periódico El Siglo, Domingo 22/10/2000

7 Delval, Juan; Enesco, Ileana. Hacer reforma moral, desarrollo y educación. Grupo Anaya. S.A : Madrid. 1998

8 Donas S: "Marco epidemiológico conceptual de la salud integral del adolescente". Versión revisada y ampliada noviembre 1997, OPS. Costa Rica . 1997.

9 Dulanto, Enrique. "El adolescente". Asociación Mexicana de Pediatría. Educación en Valores A.C., Mexico. 1989

10 Edisa. Educación a distancia en salud del adolescente. Módulo 9.La educación en la Europa de los siglos XVIII y XIX.Fundación Kellogg. Buenos Aires. 1997

11 Secretaría de Estado de Educación y Cultura. Educación en Valores, Editora Centenario, S.A. Abril 1998. Santo Domingo 112p.

12 F. Compagnoni, G. Piana y S. Privitera, M. Vidal. "Nuevo Diccionario de Teología Moral", Ediciones Paulinas 1992. Madrid, España, 1980 págs.

13 Frondizi, R., “Qué son los valores?” FCE, 5ta. Edic. México, D.F. 1972.

14 Secretaría de Estado de Educación Bellas Artes y Cultos. "Fundamentos del Curriculum (Tomo I y II)", Editora Alfa \& Omega, lera. Edic. Santo Domingo, 1994.

15 Gafo, Javier: "Los principios de justicia y solidaridad en bioética”, en: Cuadernos regional de bioética, edición $\mathrm{N}^{\circ}$ 6, agosto 1998, Santiago, Chile.

16 González Alvarez, Luis José: "Etica Latinoamericana”, Universidad Sto. Tomás- USTA, Bogotá, Colombia, 1994.

17 Gracia, Diego. Notas de cátedra magister bioética. Universidad de Chile. 1998. Chile.

18 Gracia, Diego: "Fundamentos de Bioética", Eudema, S.A., Madrid, España, 1987, 
19 Gracia Diego. «Etica de los confines de la vida». Etica y Vida N. ${ }^{\circ} 3$.El Buho.Colombia. 1998

20 Hernández Sampieri, Roberto; Fernández Collado, Carlos; Baptista Lucio, Pilar. " Metodología de la Investigación". Editora Mc GrawHill, S.A. México, D.F. 1998.

' Erickson, Erick, 1974. Identidad, Juventud y Crisis.

${ }^{2}$ Douvan y Adelson, 1980, Handbook on Adolescence. J. Willey y Sons 